\title{
Bending deformation analysis of gear hone tooth lateral faces
}

\author{
Yuriy Bagaiskov ${ }^{1, *}$ \\ ${ }^{1}$ Volzhsky Polytechnic Institute (branch) of the Volgograd State Technical University, Volzhsky, \\ Volgograd Region, Russia
}

\begin{abstract}
Gear hones are used for finish machining of hardened gear tooth lateral faces by the generating method. In service, due to penetration of abrasive grains into metal, wear and running-in of the tool material, especially in the case of elastic binding agents, the tooth contact takes place not in a point, but in an ellipse area. The total bending deformation of a hone tooth in the contact point is a sum of the fixed tooth bending deformation and the deformation, characterizing the tooth root travel in a hone rim. The calculations make it evident that hone tooth bending deformation value depends on the contact point vertical position; it drops by a factor of $400-500$ from the lower contact point to the top. Besides, deformation increases by a factor of 60 with decrease of the elasticity modulus. The rim part adjacent to a tooth is also considered during analysis of the second component of the total tooth bending deformation, characterizing the tooth root elastic strain. With the tooth height increase, this deformation value increases by a factor of $4-20$ and significantly (by an order of magnitude) increases with the elasticity modulus drop. Bending deformation analysis results of gear hone teeth are applied for studying their operation capabilities, as well as development of geometry and compound specifications.
\end{abstract}

A gear hone is an abrasive tool for finish machining of hardened gear tooth lateral faces; as a rule, it is made in the form of a helical gear and operates applying the generating method, with reverse rotation and longitudinal feed.

Theoretically, the contact of hone tooth lateral faces and a gear, when their axes intersect, takes place in a point. In practice, due to penetration of abrasive grains into metal, wear and running-in of the tool material (event with its infinite stiffness) during operation, the contact takes place along a small ellipse-shaped area [1]. As distinct from the gear mesh, a hone and a processed gear have essentially different stress-strain performance. The hone material elasticity modulus resides in a wide range of values, primarily depends on the binding agent type, from epoxy to elastic polyurethane $\left(E_{0}=0,1 \cdot 10^{3}-6 \cdot 10^{3} \mathrm{MPa}\right)$, and is $40-2000$ times lower than the material elasticity modulus of the parts machined by hones $\left(E_{1}=2,15 \cdot 10^{5} \mathrm{MPa}\right)$.

\footnotetext{
* Corresponding author: instra-ysb@,rambler.ru
} 
A hone tooth elastic model is shown in Fig. 1. $A$ and $B$ are the conventional axes of tooth turning due to bending deformation and in relation to the hone rim. The model gives a sense of all the components of gear hone tooth total deformation (except for the rim deformation) in contact point $K$ due to the stiffness of corresponding springs. The travel of spring 1 enables simulation of the contact deformation $\left(\omega_{K}\right)$, round-section spring 2 - rotation of the tooth symmetry axis proportional to the bending deformation $\left(\omega_{H}\right)$, round-section spring 3 - tooth root rotation about the rim $\left(\omega_{O C}^{\prime}\right)$, springs 4 and 5 - tooth root shifts in the directions lateral and parallel to its symmetry axis $\left(\omega_{O C}^{\prime \prime}, \omega_{O C}^{\prime \prime \prime}\right)$.

Studies showed that bending components of deformation dominate the change of hone operation travel-time parameters and hone operating results.

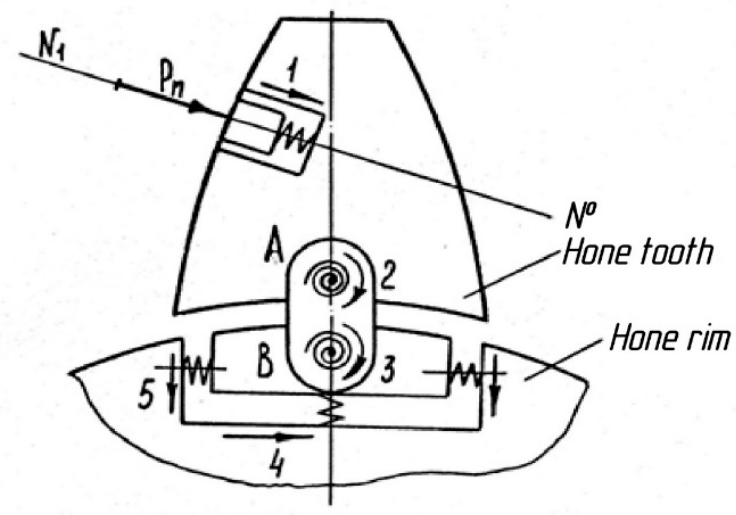

Fig.1. Gear hone tooth elastic model

The total bending deformation of hone tooth $\omega_{\Sigma u}$ in the contact point is a sum of fixed tooth bending deformation $\omega_{u}$ and the deformation, characterizing the tooth root travel in a hone $\operatorname{rim} \omega_{o c}$ :

$$
\omega_{\Sigma u}=\omega_{u}+\omega_{o c}
$$

Gear tooth bending deformation is calculated using the following formula:

$$
\begin{gathered}
\omega_{u}=\frac{2 P_{n} K_{\ni}}{E_{0} \cos ^{2} \beta_{0}}\left(K_{1}+K_{2}+K_{3}\right) \\
K_{1}=\frac{\cos ^{2} \alpha_{w}}{2 \alpha_{w}-\sin 2 \alpha_{w}}\left[\ln \frac{R_{f o}}{R_{K}}-\left(1-\frac{R_{K}}{R_{f 0}}\right)\right] ; \\
K_{2}=\frac{\sin ^{2} \alpha_{w}}{2 \alpha_{w}-\sin 2 \alpha_{w}}\left[\ln \frac{R_{f o}}{R_{K}}-\left(1-\frac{R_{K}}{R_{f 0}}\right)\right] ; \\
K_{3}=-\frac{1}{i n v 2 \alpha_{w}}\left(1-\frac{R_{K}}{R_{f 0}}\right)^{2} .
\end{gathered}
$$


Availability of slope angle $\beta_{0}$ according to the recommendations $[2,3]$ and tooth correction value $X_{0}$ are considered in formula (2). Dimensionless ratio $K_{1}$ characterizes the influence of shearing force $P_{n} \sin \gamma, K_{2}$ - of compression force $P_{n} \cos \gamma, K_{3}$ - of bending moment $P_{n} R_{f 0} \sin (\alpha+\gamma)$.

Tooth profile curvature ratio $K \ni$ addresses the involute profile replacement with a straight profile for naive simulation. Its value is taken depending on teeth number $Z_{0}$ and the studied point position along the tooth height according to the recommendations [2].

Distances $R_{K}$ and $R_{f 0}$ that determined the position of point $K$ along the hone tooth height are calculated depending on shifting ratio $X_{0}$.

Similarly to the six analyzed hone tooth profile points along its height from the bottom to the top at $K_{i}=-1,25 ;-1 ;-0,5 ; 0 ; 0,5 ; 1$ :

$$
R_{K}=\frac{\frac{\pi m}{4 \operatorname{tg} \alpha}-\frac{K_{i} m+x_{0} m}{\cos \alpha_{W}}}{\cos \alpha_{W}}=\frac{m\left[\pi \cos \alpha_{W}-4\left(K_{i}+x_{0}\right) \operatorname{tg} \alpha\right]}{4 \operatorname{tg} \alpha \cos ^{2} \alpha_{W}} .
$$

Besides, the tooth height datum $(\mathrm{O})$ is taken on the initial hone diameter.

For $K_{i}=1,25 \quad R_{K}=R_{E}=R_{f 0}$, at $K_{i}=0 \quad R_{K}=R_{W 0}$.

The arm of action load $P_{n}$ upon the hone tooth $(h)$ in the five analyzed points: $h=0.25$ $\mathrm{m} ; 0.75 \mathrm{~m} ; 1.25 \mathrm{~m} ; 1.75 \mathrm{~m} ; 2.25 \mathrm{~m}$.

The dynamic analysis results of the $\omega_{u}$ values in the contact point along the hone tooth height depending on $E_{0}$ are given in Fig. 2.

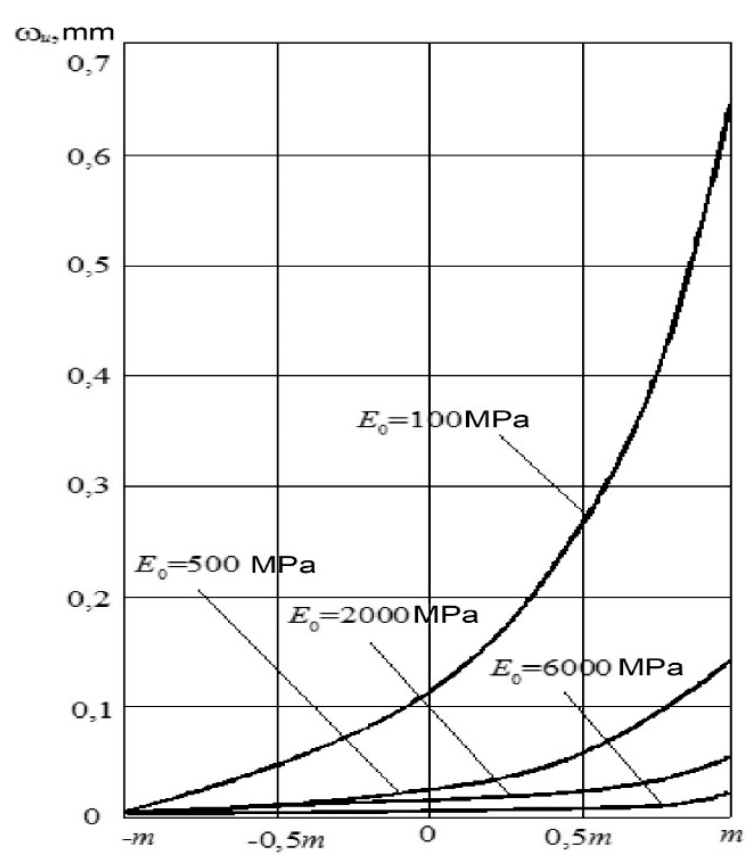

Fig. 2. Variation of bending deformation $\omega_{u}$ along the hone tooth height for various values of $E_{0}$ 
The calculations make it evident that hone tooth bending deformation value depends on the contact point vertical position; it drops by a factor of $400-500$ from the lower contact point to the top. Besides, $\omega_{u}$ increases by a factor of 60 with decrease of $E_{0}$ from 6000 $\mathrm{MPa}$ to $100 \mathrm{MPa}$.

The tooth-adjacent part of the rim with a section, equal to the tooth root width $\left(S_{f 0}\right)$, of actual distributed normal and tangential loads is considered during analysis of the second component of the total tooth bending deformation in the contact point, characterizing the tooth root elastic strain.

The depth of conventional tooth fixing in the rim depends on the rim design (a disk or a ring). The point where normal travel is 100 times less than that in the contact point is taken as the fixed point [2].

Hone tooth root elastic strain $\omega_{o c}$, as a component of total bending deformation $\omega_{\Sigma u}$, is determined using the following formula:

$$
\omega_{o c}=\frac{P_{n}}{2} \lambda_{0}[L(l)+K(\alpha, S)]
$$

wherein $\lambda_{0}-$ is the ratio, characterizing the tooth material elastic properties,

$$
\lambda_{0}=\frac{2\left(1-\mu_{0}\right)^{2}}{\pi E_{0}}
$$

wherein $L(l)$ is the root deformation component dependent on the depth of the conventional tooth embedment into the hone rim,

$$
L(l)=(1-l) \ln \left|\frac{1+l}{1-l}\right|+2 \ln \frac{\sqrt{l}}{2}|1-l|
$$

$K(\alpha, S)$ is the deformation component dependent on the gear geometry and contact point position along the tooth height,

$$
K(\alpha, S)=\left(\frac{6 h}{S_{f 0}} \cos \alpha\right)^{2}\left(1-\frac{S_{K}}{2 h} \operatorname{tg} \alpha\right)
$$

In formulas $(6)-(8): \mu_{0}$ is the hone material Poisson ratio, for polymer materials $\mu_{0}=$ 0.4 ; $l$ is the ratio, characterizing the position of the point of the conventional tooth embedment into the hone rim with null deformation; $h$ is the arm of acting force $P_{n}$ in the five analyzed points, $h=0.25 \mathrm{~m} ; 0.75 \mathrm{~m} ; 1.25 \mathrm{~m} ; 1.75 \mathrm{~m} ; 2.25 \mathrm{~m}$.

Distance $S_{K} / 2$ of each contact point to the tooth axis and the half-width of its root $S_{f 0} / 2$ at $K_{i} m=-1,25 m,-m ;-0.5 m ; 0 ; 0.5 m ; m$

are determined as follows:

$$
S_{K} / 2=m\left[\frac{\pi}{4}+x_{0} \operatorname{tg} \alpha-\left(K_{i}+x_{0}\right) \operatorname{tg} \alpha_{W}\right] .
$$

At $K_{i}=1,25 S_{K} / 2=S_{f 0} / 2$ 
The conventional tooth embedment depth was determined by analogy with tooth gears [2] using the following formula applied in case of a ring rim shape which is also common for hones:

$$
l=\frac{0,7 \Delta R}{S_{f 0}} .
$$

The dynamic analysis results of $\omega_{o c}$ along the hone tooth height depending on $E_{0}$ are given in Fig. 3.

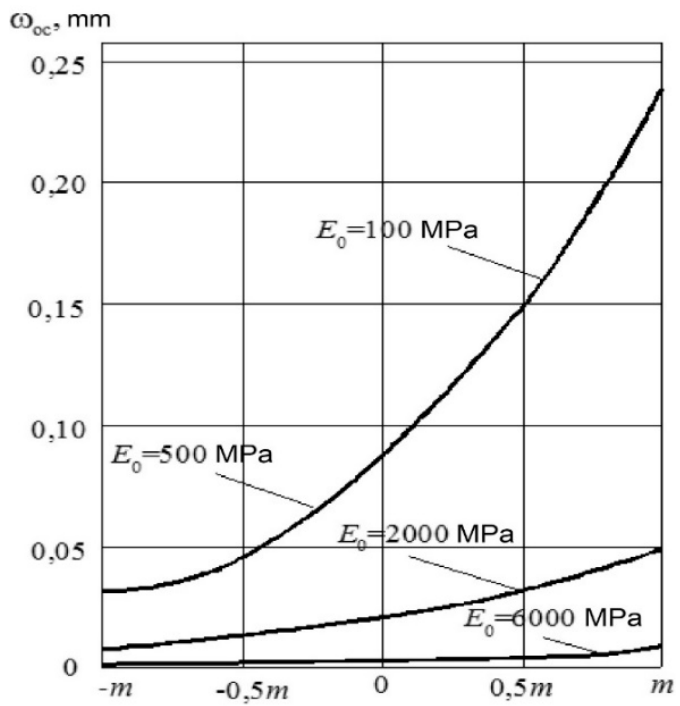

Fig. 3. Variation of $\omega_{o c}$ along the hone tooth height for various values of $E_{0}$

The value of total hone tooth bending deformation component $\omega_{o c}$ in contact point, characterizing the tooth root travel in the hone rim, increases by a factor of $4-20$ with increase of tooth height $h$ and significantly (by an order of magnitude) increases with a drop of $E_{0}$.

The results of the bending deformation analysis of gear hone teeth are confirmed by experiments and applied for studying their operation capabilities, as well as development of geometry and compound specifications [4].

\section{References}

1. Yu. Taramykin, Momentary Tooth Contact Shape and Area in Case of Gear Honing, Collection of Works by ENIMS Postgraduates, pp. 188 - 197 (1966)

2. E. Airapetov, M. Genkin, D. Kolin, Straight-Tooth Spur Gearing Flexibility, Vibroacoustic Activity of Gear-Driven Mechanisms, pp. 13 - 59 (1971).

3. K. I. Zablonsky, S. Filipovich, Tooth Stiffness Analysis of Spiral Gears, News of Higher Institutions. Engineering, c. 75 - 79(1976)

4. Yu. Bagaiskov, Peculiarities of Gear Hone Design, Abrasive Machining Processes, Abrasive Tools and Materials. Shlifabraziv - 2004, pp.69 - 70 (2004) 